\title{
Closer to nature: new biomaterials and tissue engineering in ophthalmology
}

Bruce Allan

Ophthalmology has a long history of successful conventional biomaterial applications including viscoelastics, drug delivery vehicles, contact lenses, and a variety of implants. A myriad of further possibilities exists as the margins between conventional material concepts and natural tissues continue to blur, and biomaterials move closer to nature. Genetically engineered materials (for example, hyaluronic acid and fibrin tissue glues) harnessing the power and accuracy of biological systems in molecular synthesis are becoming commonplace. New synthetic surfaces capable of upregulating or downregulating biological responses at the tissue/material interface are starting to reach clinical application; and an emerging understanding of matrix/cell interactions may soon allow engineered replacement for a range of tissues in the eye.

\section{Synthetic materials in ophthalmology}

A basic classification divides materials according to their primary bonding structure into ceramics (ionic bonding), metals (metallic bonding), and polymers (covalent bonding). Modern ophthalmic implants are almost all fabricated from synthetic polymers.

Polymeric materials are composed of long chain molecules (polymers) synthesised from repeat units (monomers) whose chemical character and reactivity determine many bulk properties. Most polymer chains have a covalently bonded backbone of carbon atoms joined to a variety of pendant groups. For siloxanes ("silicone"), an important group of synthetic biomaterials, this backbone consists of alternating atoms of silicone and oxygen. Molecular chains vary in length and are irregularly intertwined, although areas of regular arrangement (crystallinity) may exist. Cross linkage density and the density of secondary bonding further determine bulk properties for a given polymeric material. ${ }^{1}$

\section{Biological conditioning after implantation}

Secondary bonding mechanisms (for example, hydrogen bonds, van der Waals forces) are particularly relevant to biological systems, and are thought to have an important role in modulating protein conditioning - the process by which relatively inert polymeric material surfaces are rendered biologically active by contact with the tissues or body fluids. ${ }^{2}$

Protein conditioning is partly determined by surface reactivity, which varies between materials. Surface molecules tend to have more unoccupied bonding sites than molecules buried within a material, and are at a relatively higher energy state (Fig 1). Interfacial free energy for a material surface is a measure of the number of free bonding sites per unit area and their reactivity. Soluble proteins can often achieve a lower energy state by occupying these free sites, and synthetic materials are quickly coated after exposure to a biological environment. ${ }^{3}$
The pattern of protein adsorption varies between materials, and influences subsequent biological interactions. ${ }^{3}$ Soluble proteins compete for material surface bonding sites after implantation in a "race for the surface" ${ }^{4}$ Differential adsorption is determined by factors including implant surface chemistry, concentration in the fluid surround, and intrinsic surface reactivity for each protein constituent of the adsorbed film. Adsorption is sufficiently rapid that cells may never encounter an unconditioned material surface..$^{5-7}$

Changes in tertiary structure (molecular folding) occur after adsorption. Proteins are probably partially denatured but retain modified biological activity. ${ }^{37}$

\section{Foreign body inflammation}

Inflammatory cell interactions with protein conditioned surfaces are incompletely understood. ${ }^{7}$ The classic foreign body response involves adhesion of, firstly, neutrophils then macrophages to the material surface. Cytokine elaboration activates fibroblasts, and implants are walled off by a variable thickness of new collagen. Macrophages persist at the material surface in the long term, and commonly aggregate to form multinucleate giant cells. The extent to

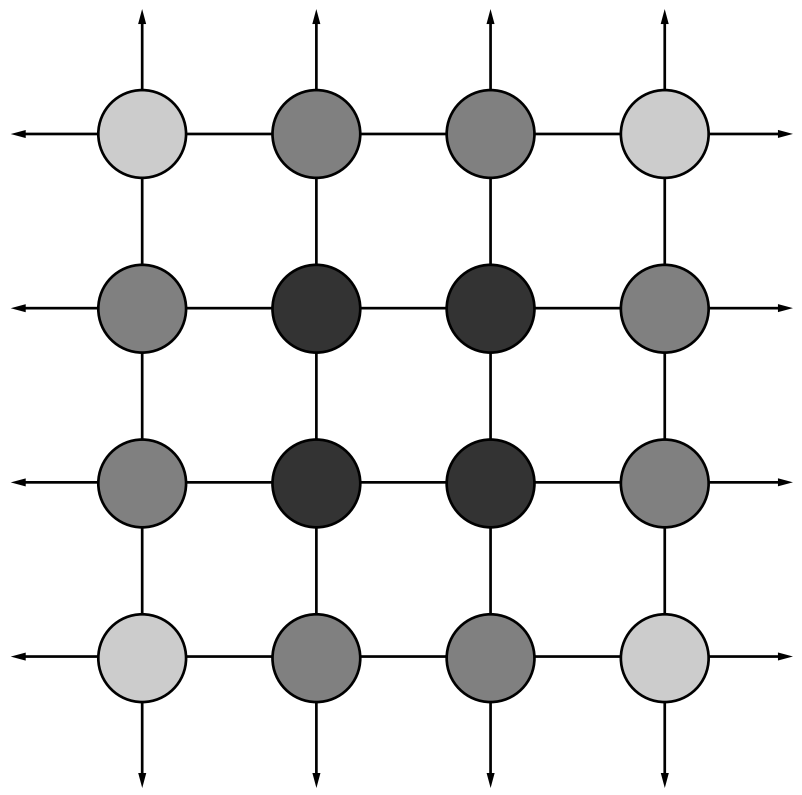

Figure 1 A simple two dimensional lattice illustrating surface reactivity. Molecules within the lattice are at a lower energy state (darker shade) than molecules at the surface which have more unoccupied bonding sites (arrows). Interfacial free energy is a measure of the number and reactivity of unoccupied bonding sites at the interface between a material surface and its surroundings. Polymers such as poly (tetrafluoroethylene) (PTFE,

Teflon) have a have a relatively unreactive surface and are less prone to biological spoilation in aqueous systems than hydrophobic materials (for example, silicone, PMMA) with a higher interfacial free energy. 

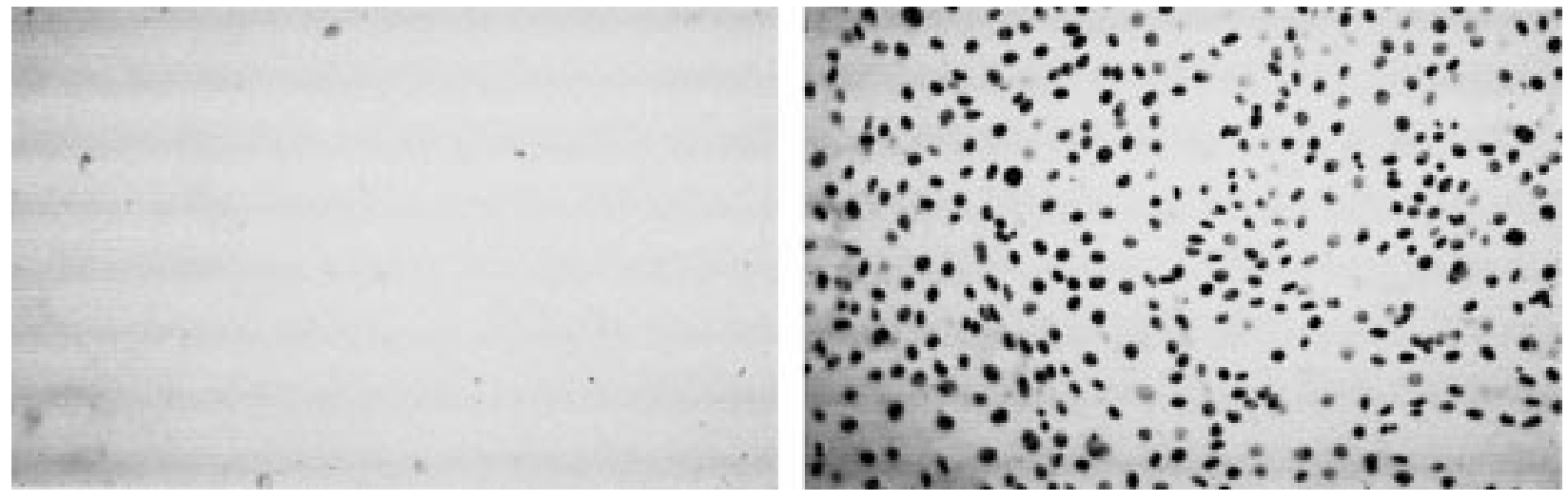

Figure $23 T 3$ fibroblasts cultured for 24 hours with bromodeoxyuridine (BRDU) to label new DNA shows cell adhesion and division is greatly reduced for phosphoryl choline copolymer coated (left) versus uncoated (right) poly (methylmethacrylate) (PMMA). (Courtesy of Dr Andrew Lloyd, University of Brighton.)

which macrophages remain active in elaborating proinflammatory cytokines is unknown. ${ }^{8}$ Some continued inflammatory activity can be inferred from progressive encapsulation responses, as with glaucoma filtration implants for example; and may be influenced by chemical (for example, material degradation) or mechanical factors (for example, implant stability and micromotion). ${ }^{78}$

An insight into the influence of protein conditioning in the initiation of inflammatory responses at material surfaces is available from animal studies examining neutrophil and macrophage accumulation on intraperitoneal implants in mice. ${ }^{910}$ Pretreating with albumin (occupying surface binding domains with a relatively inert protein) reduces inflammatory cell recruitment. Complement can be activated by adherent immunoglobulins or directly via the alternate pathway to initiate neutrophil recruitment. Both complement depleted and hypogammaglobulinaemic mice are capable of mounting a normal inflammatory response, however, indicating that neither immunoglobulins nor complement are actually required to initiate inflammation. In contrast, fibrinogen depleted mice do not mount a normal inflammatory response unless the implant is precoated with fibrinogen. Fibrinogen adhesion would therefore appear to have a pivotal role in initiating inflammatory cell recruitment. ${ }^{9}$

Cells do not behave as simple charged spheres during adhesion and spreading. Although some correlation between hydrophilicity (an index of interfacial free energy) and biological reactivity has been observed for material surfaces, independent variables including surface texture and receptor specific binding are also important. ${ }^{11}$ Fibrinogen is thought to undergo conformational changes after surface binding to reveal receptor specific domains, which encourage inflammatory cell adhesion. ${ }^{70}$

\section{Bacterial colonisation}

Another aspect of the "race for the surface" after material implantation is competition between bacteria and tissue cells for reactive domains at a conditioned surface. If bacterial colonisation is established on a synthetic surface, it is difficult to eliminate because of enhanced bacterial resistance to both antibiotics and host defence mechanisms for organisms encased in biofilm. ${ }^{4}$ Tissue integration is prevented by continued inflammation, and infection is a common cause of implant extrusion. Conversely, good tissue integration and pre-existing colonisation with eukaryotic cells tend to protect from bacterial adhesion. ${ }^{4}{ }^{12}$

\section{Biocompatibility}

Biocompatibility is a multidimensional concept, which escapes easy definition. In general, an ideal biomaterial would not induce an inflammatory response, would resist bacterial colonisation, and would promote normal differentiation in the surrounding tissues. ${ }^{13}$ Specific requirements for biocompatibility vary with the application and site of implantation, and may conflict. For stable tissue integration, surface modification to promote cell adhesion is desirable. For fluid contacting applications, the reverse is true: biocompatibility may be significantly impaired by cell adhesion.

\section{Bioinert materials}

Bioinert materials non-specifically downregulate biological responses. These materials were developed originally as non-thrombogenic surfaces for vascular surgery, and are often referred to as haemocompatible; but have a number of possible applications in the eye and other biological fluid contacting environments. In addition to reducing fibrin deposition and platelet activation, bioinert materials resist biological spoilation generally. Protein deposition, bacterial and inflammatory cell adhesion are all reduced. Potential ocular applications include contact lenses, intraocular lenses, glaucoma drainage devices, keratoprosthesis optics, and vitreous substitutes.

Successful development of synthetic bioinert materials has been derived from mimicking natural surfaces. A new group of materials has been polymerised from monomers based on phosphoryl choline, the hydrophilic head group of phospholipids (lecithin and sphingomyelin) which predominate in the outer envelope of mammalian cell membranes. ${ }^{14}{ }^{15}$ In vitro assays for a range of phosphoryl choline (PC) copolymer coated surfaces demonstrate a generalised reduction in protein and cell adhesion (Fig 2) in comparison with uncoated controls. ${ }^{16-18}$ Clinical trials in contact lens wearers show reduced protein and lipid spoilation, ${ }^{19}$ and increased comfort ${ }^{20}$ for patients wearing a PC based hydrogel lens in one eye and a conventional hydrogel lens in the other.

The mechanism by which PC polymers resist protein and cellular adhesion is the subject of continuing debate. The natural cell wall phospholipid bilayer is self assembling. Stability is achieved by sequestering hydrophobic lipid moieties to the interior in an aqueous environment. PC polymers may mimic natural cell surfaces by preferentially adsorbing a self assembling phospholipid monolayer in the correct configuration. ${ }^{21}$ Alternatively, resistance to protein and cell adhesion may be mediated by properties intrinsic to the PC molecule. ${ }^{14}{ }^{15} \mathrm{PC}$ is zwitterionic, possessing both positive and negative charges in overall electrical neutrality. This juxtaposition attracts a large and stable hydration shell, which effectively lowers interfacial free energy and the access to bonding sites for adsorption (Fig 3). 


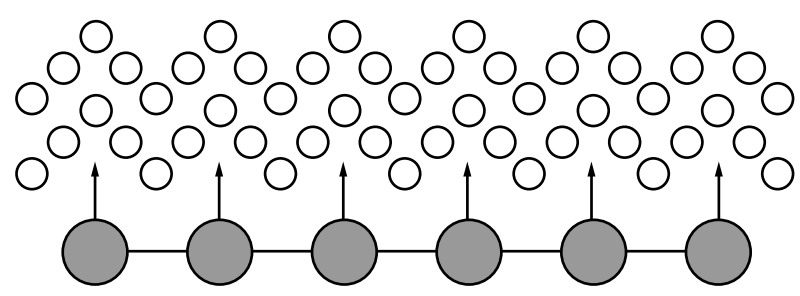

Figure 3 Hydrogels reduce their interfacial free energy in aqueous systems by trapping a shell of water molecules (open circles) which tend to shield their reactive domains (arrows). Bioinert polymers and natural cell surfaces may resist non-specific adsorption through this micromolecular exclusion zone.

The ability to trap water and exclude non-specific macromolecular adsorption may be of fundamental importance to natural tissue surfaces. Conventional synthetic hydrogels (weakly cross linked, hydrophilic, soft polymers) are generally more biologically inert than might be expected in comparison with non-hydrated polymeric materials with similar surface energy characteristics. Poly(hydroxyethylmethacrylate) (HEMA), for example, deposits cells less readily than poly(methylmethacrylate) (PMMA) when used as an intraocular lens material. ${ }^{22}$ Again, this may be explained by a reduction in available bonding sites caused by water trapping at the material surface.

Polyethylene oxide polymers (PEO) are another potentially useful group of bioinert materials, which loosely reflect some of the properties of natural mucous membrane surfaces. PEO polymers are highly hydrophilic, mobile, long chain molecules, which trap a large hydration shell. They enhance resistance to protein and cell spoilation when grafted onto a variety of material surfaces. $^{23} 24$ PEO polymers are also amenable to end group coupling for surface immobilisation of biologically active molecules (for example, heparin) to add specific functionality. ${ }^{24}$

No synthetic biomaterial surface is truly bioinert, but materials such as PC and PEO polymers would appear to offer considerable promise in downregulating some of the deleterious biological reactions associated with conventional polymeric materials in ophthalmology.

\section{Bioactive materials}

Zero reaction is often an inappropriate biological reaction to implanted materials. This is particularly true in situations where good tissue integration or tissue regeneration is paramount.

Success in creating materials which encourage cell adhesion, tissue integration, and tissue regeneration has been derived from mimicking the natural interface between hard and soft tissues. Bioactive materials upregulate specific elements of the biological response at the tissue/material interface. ${ }^{13}$ An important group of bioactive materials encourages bonding with the soft tissues through an interfacial layer of hydroxyapatite, ${ }^{25}$ the predominant mineral constituent of bone. Porous hydroxyapatite coral implants are already widely used in ophthalmology as post enucleation ball implants. ${ }^{26} \mathrm{~A}$ keratoprosthesis with a coral skirt element has also been described. ${ }^{27}$ Synthetic bioactive materials, including a range of hydroxyapatites and glass ceramics, have been developed for hard tissue replacement. They are available as coatings, resorbable gels, and ceramic-polymer composites. ${ }^{25}$ Existing applications include ossicular replacement in degenerative middle ear disease ${ }^{28}$ periodontal bone regeneration, ${ }^{29}$ and orbital floor repair. $^{30}$

Hydroxyapatite based bioactive implants are thought to promote normal differentiation in surrounding tissues by providing an enhanced environment for cell adhesion. ${ }^{25}$
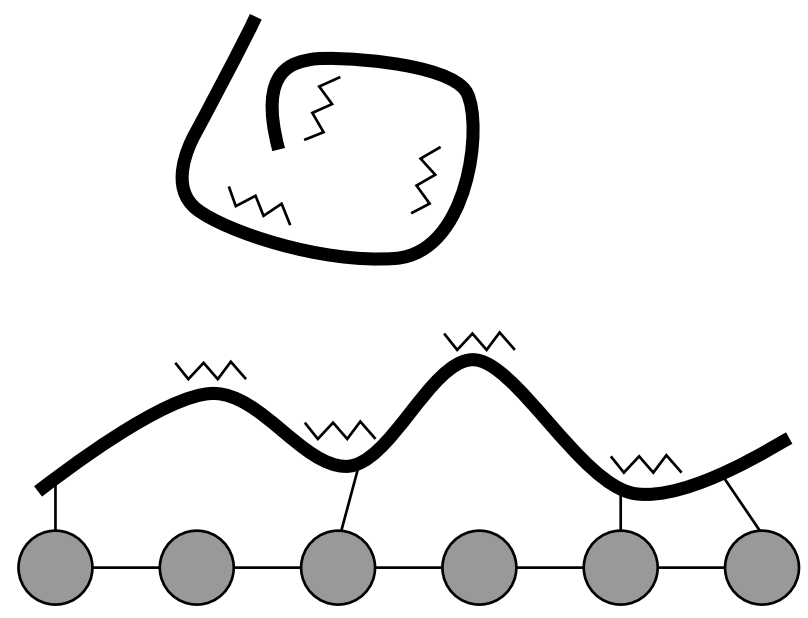

Figure 4 Adsorption of soluble adhesion molecules (thick lines) to a hydroxyapatite surface may induce a conformational change resulting in the exposure of previously sequestered integrin binding domains (jagged lines), promoting cell adhesion. The effect of incorporating integrin-ligand-peptide sequences on cell behaviour in synthetic matrices and artificial surfaces is currently being explored.

Cytoskeletal microfilaments (for example, actin, myosin, actinin, and tropomyosin) controlling cell shape and migration are coupled through specialised cell membrane proteins (integrins) to extracellular adhesion molecules (for example, fibronectin, laminin, vitronectin, thrombospondin) present in basement membranes and tissue matrices. ${ }^{31} 32$ An interfacial layer of hydroxyapatite may adsorb adhesion molecules in a favourable configuration (Fig 4), exposing a high density of integrin ligand domains. $^{33}$ This promotes the formation of focal adhesions (Fig 5). Without these anchoring points, cells are unable to express a normal phenotype or respond to trophic cytokines during surface or matrix colonisation. ${ }^{35} 36$ Growth factors may also be adsorbed to hydroxyapatite surfaces in a favourable configuration, further promoting tissue integration..$^{25} 37$

Current efforts are being directed towards distilling the essence of cell adhesion requirements in new biomaterials incorporating minimal peptide sequences from the adhesion molecules responsible for integrin binding. The best characterised of these integrin ligand domains is the RGD sequence (arginine-glycine-aspartate) present in fibronectin, vitronectin, collagen, and laminin. ${ }^{31}$ Polymer matrices incorporating RGD sequences enhance cell integration. ${ }^{38} \mathrm{~A}$ variety of integrins bind to the RGD sequence. Enhanced cell adhesion is relatively nonspecific, and inflammatory cell binding may compete with regenerative cell populations for the available binding domains. With the incorporation of more selective integrin binding sequences, it may be possible to further encourage regenerative responses at the expense of inflammation and wound healing. ${ }^{39}$

\section{Tissue engineering}

Beyond simply improving tissue integration for synthetic implants, functional tissue regeneration within artificial matrices or on artificial surfaces is now possible. This is where the new science of tissue engineering diverges from conventional biomaterials research. Tissue engineering combines elements of engineering and materials science with genetics, molecular, cell, and developmental biology in organ replacement and organ regeneration..$^{40}{ }^{41}$ Engineered replacement tissue constructs are already in development for a variety of tissues including skin, cartilage, nerve, liver, kidney, muscle, heart valves, and blood vessels. ${ }^{42-50}$ 


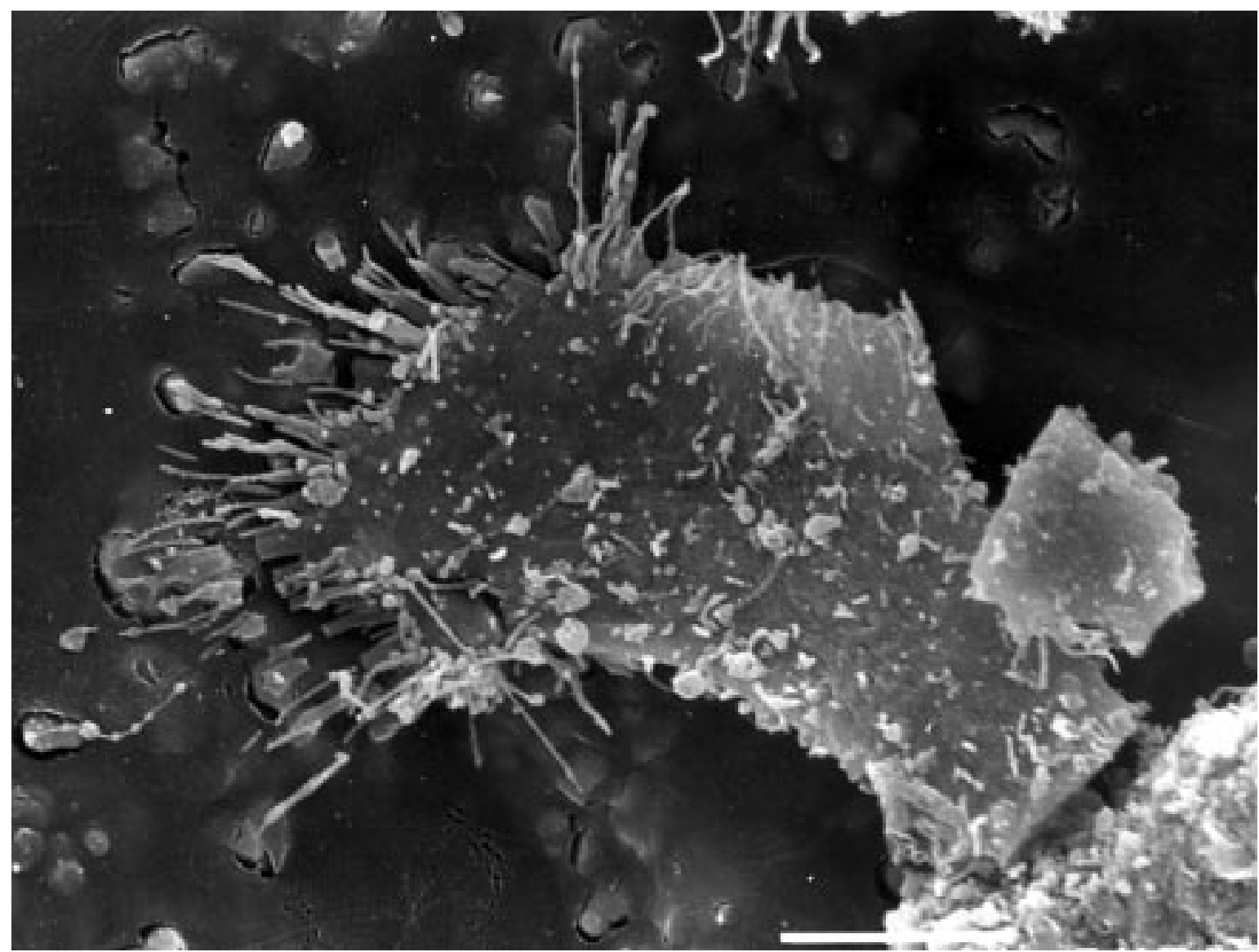

Figure 5 A scanning electron micrograph showing a human osteoblast reaching pseudopodia out to hydroxyapatite particles (lighter areas) dispersed within a bioactive ceramic-polymer composite (HAPEX) (scale bar $=10 \mu \mathrm{m}$ ). (Courtesy of Dr Lucy Di Silvio, The IRC in Biomedical Materials, University of London.)

Many organs have regenerative capacity and will regenerate rather than scar in the absence of matrix destruction. Good examples include liver, lung, and epithelial surfaces throughout the body including skin and the ocular surface. One strategy in tissue engineering with possible relevance to ophthalmology is the replacement of damaged tissue with engineered matrices to restore a normal cell adhesion environment. Good regenerative responses have been observed clinically after extensive burns using artificial skin constructs based on collagen/proteoglycan coprecipitates. ${ }^{51}$ Enhanced axonal regeneration has also been demonstrated in a rat model, ${ }^{45}$ in which a portion of the sciatic nerve is replaced by a similar collagen/proteoglycan coprecipitate within a collagen tube. These matrices are degraded and replaced by autologous matrix from regenerating cells. Collagen cross linkage density is varied to match the rate of matrix degradation with the rate of healing for the tissue to be regenerated. Pore size and directionality are also controlled to optimise results in the target tissue. ${ }^{52}$

In some circumstances it may be desirable to preseed the matrix with donor cells in order to normalise the initial cell signalling environment, rather than waiting for autologous cells to populate an engineered matrix. For epithelial surfaces, the key to regeneration appears to be a normalised substrate. In skin, for example, dermal replacement promotes epidermal regeneration. Allogeneic dermal fibroblasts are only weakly antigenic. Neonatal foreskins, discarded at circumcision are used as a source of fibroblasts for cell seeded artificial skin constructs. These young cells have immense replicative potential. Incredibly, an area of artificial skin construct the size of a football pitch can be seeded from a single donor foreskin. ${ }^{53}$ Cells seeded within a collagen matrix produce proteoglycans, adhesion molecules, and growth factors when the matrix is enriched before use. Clinical trials of these skin constructs in conditions of retarded healing (diabetic foot ulcers) appear to indicate that cell viability within the engineered replacement dermis is an important determinant of successful regeneration. ${ }^{53}$

Resorbable matrices for ocular surface regeneration analogous to current artificial skin constructs may have applications in external disease, refractive surgery, oculoplastics, and glaucoma.

Progressing from essentially two dimensional constructs (for example, skin or conjunctival replacement) to solid organ replacement requires careful consideration of the nutrient environment. Most cells are unable to survive in a matrix at greater than approximately $500 \mu \mathrm{m}$ from a diffusible nutrient source (blood, aqueous, synovial or cerebrospinal fluid)..$^{54}$ This limitation for non-vascular tissues immediately suggests the cornea as a realistic target for tissue engineered replacement. Perfusion culture systems, or "bioreactors", developed for seeding artificial cartilage matrices could be modified and applied to the development of a true replacement cornea. Early studies have already demonstrated normal morphology and expression of phenotypic markers for engineered corneal constructs (Fig 6) with an epithelial and endothelial layer. ${ }^{55}{ }^{56}$ Significant problems relating to source materials and the optimisation of matrix clarity remain; but at the current rate of progress, conventional corneal transplantation may be obsolete within quarter of a century. Theoretical advantages of tissue engineered corneal replacement could include no tissue supply problems, no rejection, and no iatrogenic disease transmission. 


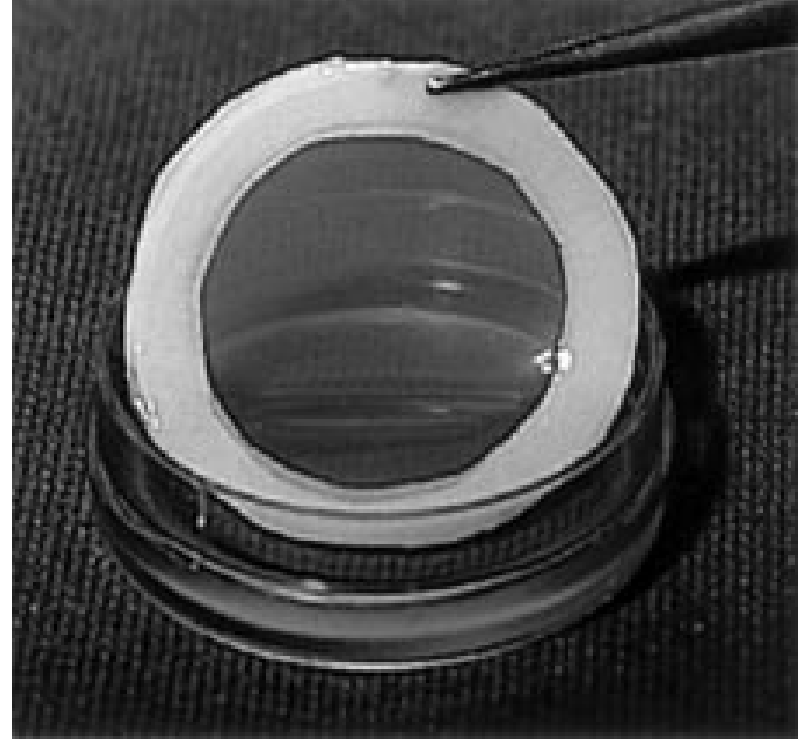

Figure 6 A tissue engineered artificial cornea. ${ }^{55}$ Sheets of keratocytes cultured for 35 days produce a thick collagenous matrix. Two of these matrix/cell sheets are then peeled away from the culture dish, and superimposed to form a corneal stromal equivalent. In appropriate conditions, a multilayered corneal epithelium can be cultured on this stromal equivalent. These epithelial cells lay down a basement membrane containing type IV collagen, lamenin, and fibronectin. (Courtesy of Dr Patrick Carrier, LOEX Laboratory, Laval University, Quebec, Canada.)

\section{Improving on nature?}

Collagen for artificial tissue matrices is currently derived from animal sources. Mammalian collagen alters little between species. Processing techniques are available to reduce antigenicity, but prion disease remains a significant concern. It is likely that recombinant collagen sources will soon be available. Synthetic resorbable matrices incorporating integrin ligand peptide sequences are also in development. ${ }^{41}$

Whilst the concept of a resorbable matrix replaced by autologous tissue is seductive in its "ultimate biocompatibility", resorbable matrices to guide tissue regeneration could also have disadvantages. The final result will leave the tissue, at best, no less resistant to injury or any underlying disease process than before the original insult. It may be possible to improve tissue performance using a permanent synthetic matrix. An artificial cornea, for example, could have tailored refractive power in addition to enhanced resistance to enzymatic matrix degradation.

Rapid evolution in cell sources for artificial tissue proceeds in tandem with advances in matrix engineering. Where regenerative potential is lost, or did not originally exist, cloned autologous tissue derived from embryonic stem cells ${ }^{57}$ may be available. Reprogramming of adult stem cells may also be possible. ${ }^{58}$

Stem cell reprogramming and cloning techniques avoid tissue rejection by producing autologous or genetically identical cell populations for tissue replacement. An alternative tissue engineering strategy with some exciting potential ocular applications is immunoisolation, in which foreign cells are protected from immune attack by encapsulation within a porous membrane ${ }^{59}$; with a pore size large enough to allow permeability to nutrients and smaller molecular species but small enough to prevent immunoglobulin and immunological effector cell access. Long term survival of allogeneic human and animal cells has been demonstrated for encapsulated cell/matrix constructs. ${ }^{59}{ }^{60}$ Current uses include liver support devices and gene therapy. In contrast with other gene therapy protocols, delivery of an engineered protein product can be measured before implantation for encapsulated cells. Sus- tained intraocular delivery of a variety of cytokines could potentially be achieved using immunoisolation technology.

Biomaterials research spans the full spectrum of possibilities for restoring tissue function from entirely synthetic, non-degradable implants and prostheses, through hybrid cell/matrix constructs, to fully resorbable matrix templates for organ regeneration. Developments throughout this exciting spectrum will change the landscape of medical practice in the coming century. The immediate challenge for ophthalmology is to translate existing bioinert, bioactive, and tissue engineering biomaterial concepts into applications relevant to the prevention of visual loss.

BRUCE ALLAN

Moorfields Eye Hospital, City Road, London EC1V 2PD

1 Strong AB. Polymeric materials. In: Strong AB, ed. Plastics: materials and processing. New Jersey: Prentice-Hall, 1996:21-92.

2 Van Wachem PB, Beugeling T, Feijen J, et al. The role of van der Vaals forces and hydrogen bonds in hydrophobic interactions between biopolymers and low energy surfaces. Interface Sci 1986;111:378-90.

3 Horbet TA. Proteins: structure, properties, and adsorption to surfaces. In: Rattner BD, Hoffmen AS, Schoen FJ, Lemons JF, eds. Biomaterials science. San Diego: Academic Press, 1996:133-41.

4 Gristina AG. Biomaterial-centred infection:microbial adhesion versus tissue integration. Science 1987;237:1588-95.

5 Schakenraad JM. Cells: their surfaces and interactions with materials. In: Rattner BD, Hoffmen AS, Schoen FJ, Lemons JF, eds. Biomaterials science. San Diego: Academic Press, 1996:141-7.

6 Strong AB, Stubley GD, Chang G, et al. Theoretical and experimental analysis of cellular adhesion to polymer surfaces. F Biomed Mater Res 1987; 21:1039-55.

Tang L, Eaton JW. Inflammatory responses to biomaterials. Am f Clin Pathol 1995;103:466-71.

8 Anderson JM. Inflammation, wound healing and the foreign body response. In: Rattner BD, Hoffmen AS, Schoen FJ, Lemons JF, eds. Biomaterials science. San Diego: Academic Press, 1996:165-73.

9 Tang L, Eaton JW. Fibrinogen mediates acute inflammatory responses to biomaterials. F Exp Med 1993;178:2147-56.

10 Tang L, Lucas AH, Eaton JW. Inflammatory responses to implanted biomaterials: role of surface-adsorbed immunoglobulin G. 7 Lab Clin Med 1993;122:292-300.

11 Ratner BD. Correlation of material surface properties with biological responses. In: Rattner BD, Hoffmen AS, Schoen FJ, Lemons JF, eds. Biomaterials science. San Diego: Academic Press, 1996:445-50.

12 Gristina AG, Naylor PT. Implant associated infection. In: Rattner BD, Hoffmen AS, Schoen FJ, Lemons JF, eds. Biomaterials science. San Diego: Academic Press, 1996:205-14.

13 Willliams DF. Definitions in biomaterials. Amsterdam: Elsevier 1987:67-8.

14 Hayward JA, Chapman D. Biomembrane surfaces as models for polymer design: the potential for haemocompatibility. Biomaterials 1984;5:135-42.

15 Durrani A, Hayward JA, Chapman D. Biomembranes as models for polymer surfaces. Biomaterials 1986;7:121-5.

16 Ishihara K, Ziats NP, Tierney BP, et al. Protein adsorption from human plasma is reduced on phospholipid polymers. F Biomed Mater Res 1991;25: 1397-407.

17 Campbell EJ, O'Byrne V, Stratford PW, et al. Biocompatible surfaces using methacryloylphosphorylcholine laurylmethacrylate copolymer. ASIAO f 1994;40:853-7.

18 Denyer SP, Lim KS, Wong L, et al. In vitro ocular compatibility of glaucoma filtration implant materials. (ARVO Suppl) Invest Ophthalmol Vis Sci 1998; 39:5114.

19 Young G, Bowers R, Hall B, et al. Clinical comparison of Omafilcon A with four control materials. CLAO f 1997;23:249-58.

20 Young G, Bowers R, Hall B, et al. Six month clinical evaluation of a biomimetic hydrogel contact lens. CLAO f 1997:23:226-36.

21 Ishihara K, Oshida H, Yutaka E, et al. Haemocompatibility of human whole blood on polymers with a phospholipid polar group and its mechanism. $\mathcal{F}$ Biomed Mater Res 1992;26:1543-52.

22 Amon M, Menapace R. Cellular invasion on hydrogel and poly(methylmethacrylate) implants; an in vivo study. $\mathcal{F}$ Cataract Refract Surg 1991;17:774-9.

23 Nagaoka S, Mori Y, Takiuchi $\mathrm{H}$, et al. Interaction between BLLD components and hydrogels with polyoxyethylene chains. In: Shalaby SW, Hoffman A, Ratner BD, Horbet TA eds. Polymers as biomaterials. New York: Plenum 1984:361-74.

24 Kim SW. Nonthrombogenic treatments and strategies. In: Rattner BD, Hoffmen AS, Schoen FJ, Lemons JF, eds. Biomaterials science. San Diego: Academic Press, 1996:297-308.

25 Cao W, Hench LL. Bioactive materials. Ceramics Int 1996;22:493-507.

26 Ashworth JL, Rhatigan M Sampath R, et al. The hydroxyapatite orbital implant:a prospective study. Eye 1996;10:29-37.

27 Leon CR, Barraquer J1 Jr, Barraquer Jl Sr. Coralline hydroxyapatite keratoprosthesis in rabbits. F Refract Surg 1997;13:74-8.

28 Dornhoffer JL. Hearing results with the Dornhoffer ossicular replacement prosthesis. Laryngoscope 1998;108:531-6.

29 Zarnet JS, Darbar UR, Griffiths GS, et al. Particulate bioglass as a grafting material in the treatment of periodontal intrabony defects. 7 Clin Periodontol 1997;24:410-18.

30 Tanner KE, Downes RN, Bonfield W. Clinical applications of hydroxyapatite reinforced materials. British Ceramic Trans 1994;93:104-7.

31 Elner SG, Elner VM. The integrin superfamily and the eye. Invest Ophthalmol Vis Sci 1996;37:696-701.

32 Hynes RO. Integrins: versatility, modulation, and signalling. Cell 1992;69: 11-25. 
33 Okamoto K, Matsuura T, Hosokawa R, et al. RGD peptides regulate the specific adhesion of osteoblasts to hydroxyapatite but not titanium. $7 \mathrm{Den}$ specific adhesion of ost
tal Res 1998;77:481-7.

34 Garcia AJ, Ducheyne P, Boettiger D. Effect of surface reaction stage on fibronectin mediated adhesion of osteoblast like cells to bioactive glass. $\mathcal{F}$ Biomed Mater Res 1998;40:48-51.

35 Schwartz MA, Schaller MD, Ginsberg MH. Integrins: emerging paradigms of signal transduction. Annu Rev Cell Devel Biol 1995;11:549-99.

36 Lauffenburger DA, Horwitz AF. Cell migration: a physically integrated molecular process. Cell 1996;84:359-69.

37 Lobel KD, Hench LL. In vitro adsorption and activity of enzymes on reaction layers of bioactive glass substrates. F Biomed Mater Res 1998;39:575-9.

38 Harrison D, Johnson R, Tucci M, et al. Interaction of cells with UHMWPE impregnated with the bioactive peptides RGD, RGE, or poly-L-lysine. Biomed Sci Instrumentation 1997;34:41-6.

39 Dee KC, Andersen TT, Bizios R. Design and function of novel osteoblastadhesive peptides for chemical modification of biomaterials. 7 Biomed adhesive peptides for chemi
Mater Res 1998;40:371-7.

40 Langer RS, Vacanti JP. Tissue engineering. Science 1993;260:920-6.

41 Langer RS, Vacanti JP. Tissue engineering: the challenges ahead. Sci Am 1999;280:62-5.

42 Bell E, Ehrlich HP, Buttle DJ, et al. Living tissue formed in vitro and accepted as skin-equivalent tissue of full thickness. Science 1981;211:1052-

43 Yannas IV, Burke JF, Orgill DP, et al. Wound tissue can utilise a polymeric template to synthesise a functional extension of skin. Science 1982;215:1746.

44 Cao Y, Vacanti JP, Paige KT, et al. Transplantation of chondrocytes utilizing a polymer-cell construct to produce tissue engineered cartilage in the shape of a human ear. Plast Reconstruct Surg 1997;100:297-302.

45 Yannas IV, Orgill DP, Silver J, et al. Regeneration of sciatic nerve across a $15 \mathrm{~mm}$ gap by use of a polymeric template. In: Gebeleim CG, ed. Advances in biomedical polymers. New York, 1987:1-9.

46 Davis MW, Vacanti JP. Towards the development of an implantable tissue engineered liver. Biomaterials 1996;17:365-72.

47 Humes HD. Tissue engineering of a bioartificial kidney: a universal donor organ. Transplant Proc 1996;28:2032-5.
48 Okano T, Matsuda T. Tissue engineered skeletal muscle:preparation of highly dense, highly oriented hybrid muscle tissues. Cell Transplant 1998;7: $71-82$.

49 Shinoka T, Ma PX, Shum-Tim D, et al. Tissue engineered heart valves: autologous valve leaflet replacement study in a lamb model. Circulation 1996;94:164-8.

50 L'Heureux N, Paquet S, Labbe R, et al. A completely biological tissue engineered human blood vessel. FASEB f 1998;12:47-56.

51 Kane JB, Tompkins RG, Yarmush ML, et al. Burn dressings. In: Rattner BD, Hoffmen AS, Schoen FJ, Lemons JF, eds. Biomaterials science. San Diego: Academic Press, 1996:360-70.

52 Yannas IV. Natural materials. In: Rattner BD, Hoffmen AS, Schoen FJ, Lemons JF, eds. Biomaterials science. San Diego: Academic Press, 1996:8494.

53 Naughton G, Mansbridge J, Gentzkow G. A metabolically active human dermal replacement for the treatment of diabetic foot ulcers. Artif Org 1997;21:1203-10

54 Sittinger M, Bujia J, Rotter N, et al. Tissue engineering and autologous transplant fromation: practical approaches with resorbable biomaterials and new culture techniques. Biomaterials 1996;17:237-42

55 Germain L, Carrier P, Giasson M, et al. A new method for in vitro production of human reconstrcuted cornea by tissue engineering. (ARVO Suppl) Invest Ophthalmol Vis Sci 1999;40:1745.

56 Cornelius EJ, Hubel A. Behaviour of corneal epithelial, endothelial, and stromal cells cultured on a three-dimensional matrix. (ARVO Suppl) Invest Ophthalmol Vis Sci 1998;39:3500.

57 Keller G, Snodgrass HR. Human embrionic stem cells: the future is now. Nat Med 1999;5:151-2.

58 Bjornson CRR, Reitze RL, Reynolds BA, et al. Turning brain into blood: a haemopoetic fate adopted by adult neural stem cells in vivo. Science 1999; 283:534-8.

59 Lysaght MJ, Aebischer P. Encapsulated cells as therapy. Sci Am 1999;280:52-8.

60 Lysaght MJ, Aebischer P. Immunoisolation and cellular xenotransplantation. Xeno 1995;3:43-8. 